\title{
Controlled Warm Working: Possible Tool for Optimizing Stored Energy Advantage in Deformed $\gamma$-fiber (ND//111))
}

\author{
R. KHATIRKAR, ${ }^{1)}$ L. A. I. KESTENS, ${ }^{2)}$ R. PETROV ${ }^{3)}$ and I. SAMAJDAR ${ }^{4)}$ \\ 1) Department of Metallurgical and Materials Engineering, Visvesvaraya National Institute of Technology, South Ambazari \\ Road, Nagpur-440011, India. 2) Delft University of Technology, Materials Science and Engineering Department, \\ Mekelweg 2, 2628 CD Delft, Netherlands. 3) Department of Metallurgy and Materials Science, Ghent University, \\ Technologiepark 903, Ghent B-9052, Belgium. \\ 4) Department of Metallurgical Engineering and Materials Science, IIT
} Bombay, Mumbai-400076, India.

(Received on August 18, 2008; accepted on October 14, 2008)

\begin{abstract}
In the present study, ultra low carbon steel samples were deformed in near plane-strain mode with different strains, strain rates and temperatures. Estimates of in-grain misorientation developments were obtained

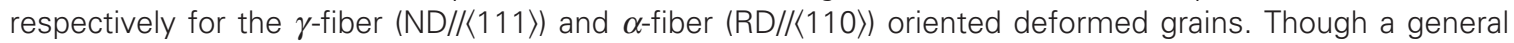
drop in in-grain misorientation was observed with increase in deformation temperature, the highest reduction of $70 \%$ showed a clear increase at the intermediate deformation temperatures. Under these condition(s), the misorientation increase in deformed $\gamma$-fiber grains was more substantial than in $\alpha$-fiber grains. The phenomenon was related to the preferred appearance of grain interior strain localizations. The study brings out a clear possibility of optimizing the stored energy advantage in the deformed $\gamma$-fiber through controlled warm working.
\end{abstract}

KEY WORDS: deformation; misorientation; microtexture; stored energy; EBSD; strain localizations; warm working.

\section{Introduction}

The ideal forming grade steel needs to satisfy two conflicting requirements - high formability and high strength. ${ }^{1-10)}$ Presence of free interstitials (such as carbon and nitrogen) is detrimental to formability but is essential for higher strengths. ${ }^{1,2,9,10)}$ Compromises are often sought through tailoring the chemistry, examples range from different grades of deep drawing quality to re-phosphorized and bake-hardening grades, ${ }^{10)}$ but rarely through serious modifications in the thermo-mechanical processing.

The deep drawability, which represents a crucial forming mode, could be characterized by the normal anisotropy or the Lankford value $r$, representing width-to-thickness strain in a tensile test. ${ }^{10)}$ The $r$ values ${ }^{1)}$ strongly depend on the crystallographic texture $^{1-3)}$ and usually display different values when measured in different directions with respect to the sample rolling direction (e.g. $r_{0}, r_{90}$ and $\left.r_{45}\right)$. The other important factor is in-plane anisotropy or $\Delta r$, where $\Delta r=\left(r_{0}+r_{90}-2 r_{45}\right) / 2$. Near zero value of $\Delta r$ is a prerequisite for the absence of 'earing ${ }^{1-3,10)}$ during deep drawing, whereas high values of the average normal anisotropy $r_{\mathrm{m}}$ $\left(r_{\mathrm{m}}=\left(r_{0}+2 r_{45}+r_{90}\right) / 4\right)$ ensure excellent deep drawability. ${ }^{1-10)}$ For BCC steel, the double boundary condition of maximizing $r_{\mathrm{m}}$ and minimizing $\Delta r$ can ideally be complied by an ideal $\gamma$-fiber $(\{111\}$ or $(\mathrm{ND} / /\langle 111\rangle))$ texture, while $\{001\}$ and $\langle 110\rangle / / \mathrm{RD}(\alpha$-fiber) are harmful to deep-drawability. ${ }^{1,2,9,10)}$ This is obtained typically through large cold re- ductions followed by recrystallization annealing - a process reported $^{2,4-10)}$ to enhance the desirable $\gamma$-fiber $(\mathrm{ND} / /\langle 111\rangle)$ with concurrent elimination of the harmful fibers $(\alpha$-fiber and cube orientations).

Formation of the $\gamma$-fiber recrystallization texture in low carbon steels ${ }^{1,2,4-14)}$ is attributed to preferential nucleation $^{2,4-6)}$ and growth ${ }^{7,9,15,16)}$ of recrystallized $\gamma$-fiber grains from the deformed grains/bands of similar orientations. Though controversies remain on the relative role of preferred nucleation and selective growth, both are usually 'linked ${ }^{\text {17-22) }}$ with preferred appearance of grain interior strain localization in near $\gamma$-fiber oriented deformed grains. In the meso-scale, such strain localizations are seen as high angle boundaries inclined at an approximate angle of $37-40^{\circ}$ with the rolling direction. ${ }^{18-20,22,23)}$ Their nature, in the micro-scale, has been reported ${ }^{19)}$ as first generation or non-crystallographic micro-bands.

Formation of strain localizations or plastic instabilities is often related to suitable instability criteria. ${ }^{24-26)}$ Dillamore's plastic instability criterion ${ }^{24)}$ is a classical example.

$$
\begin{aligned}
\frac{1}{\sigma}\left(\frac{d \sigma}{d \varepsilon}\right) & =\frac{n}{\varepsilon}+\frac{m}{\dot{\varepsilon}}\left(\frac{d \dot{\varepsilon}}{d \varepsilon}\right)+\frac{1+n+m}{M}\left(\frac{d M}{d \varepsilon}\right)-\frac{m}{\rho}\left(\frac{d \rho}{d \varepsilon}\right) \\
& \leq 0
\end{aligned}
$$

where $\sigma$ and $\varepsilon$ are the macroscopic stress and strain, $n$ and $m$ are strain hardening exponent and strain rate sensitivity, 
$\dot{\varepsilon}$ is the strain rate, $M$ is the Taylor factor and $\rho$ is the mobile dislocation density.

Negative values of $(d M / d \varepsilon)$, i.e. textural softening, is expected (and reported ${ }^{19,24-27)}$ ) to ease the formation of strain localizations provided dislocation softening $(d \rho / d \varepsilon)$ is relatively insignificant. This is achieved in low solute alloys, such as low carbon steels. Whether or not a similar effect is achievable through controlled warm or hot working has never been tested systematically. This has been the motivation for the present study. The study involved: (I) plane strain deformation (deformed at different temperatures, strains and strain rates) of ULC (ultra low carbon) steel samples and (II) detailed EBSD (electron backscattered diffraction) characterization. The later was aimed at bringing out possible effects of deformation conditions on the microstructural developments in near $\gamma$-fiber and $\alpha$-fiber oriented deformed bands/grains.

\section{Experimental Methods}

Hot rolled ULC steel, with the chemical composition given in Table 1, was subjected to plane strain compression tests. The aim of a plane strain compression (PSC - the process of rolling approximates PSC) is to compress along ND (normal direction) and to elongate, by the same quantity, along RD (rolling direction). In controlled laboratory tests, PSC can be achieved by strip PSC and by channel-die compression. ${ }^{10)}$ In the present study, strip PSC was used in a deformation simulator. The heated sample(s) was placed between two heated dies and then compressed-friction between die and sample restrained (and adequate care ${ }^{10)}$ was taken for this) material flow along TD. Two strain rates $(0.1$ and $\left.1 \mathrm{~s}^{-1}\right)$, three strains $(40,60$ and $70 \%$ reductions) and four different temperatures $\left(20,200,400\right.$ and $\left.600^{\circ} \mathrm{C}\right)$ were used - a total of 24 different deformation conditions. Samples were quenched immediately after deformation. ${ }^{* 1}$ Af-

Table 1. Chemical composition (in wt $\%$ alloying elements) of the ULC steel used in the present study.

\begin{tabular}{|c|c|c|c|c|c|c|c|}
\hline $\mathrm{C}$ & $\mathrm{Mn}$ & $\mathrm{S}$ & $\mathrm{P}$ & $\mathrm{Si}$ & $\mathrm{Al}$ & $\mathrm{Ti}$ & $\mathrm{N}$ \\
\hline 0.0014 & 0.145 & 0.0002 & 0.001 & 0.022 & 0.029 & $<0.001$ & 0.0017 \\
\hline
\end{tabular}

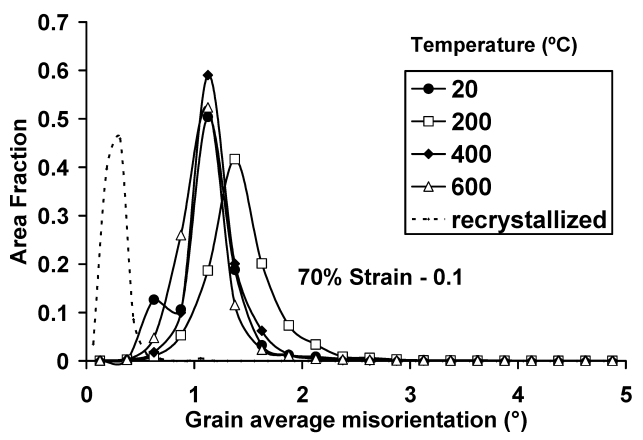

(a) terwards, the deformed samples were subjected to detailed EBSD characterization. The EBSD data were acquired in the mid-thickness section of the long-transverse plane (plane containing rolling and normal directions). EBSD samples were prepared by electro-polishing at $-20^{\circ} \mathrm{C}$ using methanol+perchloric acid electrolyte $(80: 20)$ at 11 volts DC.

A TSL-EDX EBSD system on a FEI Quanta-200 SEM (scanning electron microscope) was used for the present study. For each sample, a total area of $2 \times 2 \mathrm{~mm}^{2}$ was covered in 4 individual scans. All scans were made under identical beam and video conditions and at step size of $0.5 \mu \mathrm{m}$. For subsequent data analysis, measured points of questionable accuracy (below 0.05 confidence index ${ }^{28}$ ) were disregarded.

In-grain misorientation developments can be viewed in terms of developments in point-to-point misorientation or grain average misorientation (GAM). The GAM represents average misorientation between all neighboring pairs of points (i.e. measurement points) in a grain. The analysis of EBSD data involved two different approaches: partitioning into the so-called $\gamma$-fiber $(\mathrm{ND} / /\langle 111\rangle)$ and $\alpha$-fiber $(\mathrm{RD} / /\langle 110\rangle)$ grains and manual cropping of grains/bands. In the first approach, data/orientations within $15^{\circ}$ of the idealized fibers were isolated/partitioned and then used to estimate the GAM. The second approach, details and rationale behind, is explained subsequently in Sec. 3 (i.e. Results).

\section{Results}

In-grain misorientation development is an important aspect of plastic deformation. ${ }^{29-40)}$ Such misorientation developments can be linked to statistical estimates of GAM or average point-to-point misorientation inside a deformed grain.*2 GAM distributions at different working temperatures, for samples subjected to $70 \%$ deformation at strain rate of $0.1 \mathrm{~s}^{-1}$, are shown in Fig. 1 - for $\gamma$-fiber $(\mathrm{ND} / /\langle 111\rangle)$ and $\alpha$-fiber $(\mathrm{RD} / /\langle 110\rangle)$ grains/orientations. Also included in the figure is the GAM distribution for a fully recrystallized sample. The later can be taken as an indicator of 'measurement uncertainty'. ${ }^{41)}$ Figure 1 shows a clear shift

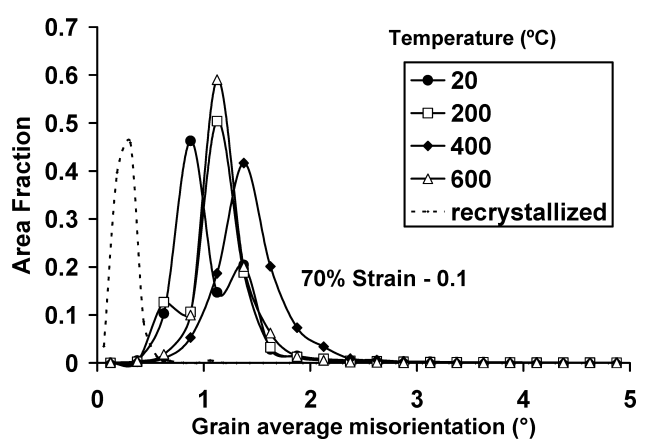

(b)

Fig. 1. GAM (grain average misorientation) distributions for (a) $\gamma$-fiber and (b) $\alpha$-fiber grains/orientations. Samples were deformed to $70 \%$ strain under a strain rate of $0.1 \mathrm{~s}^{-1}$. The GAM distribution of a typical recrystallized sample is also given for reference.

\footnotetext{
${ }^{* 1}$ It needs to be noted that the deformed samples did not show any evidence of recrystallization.

${ }^{*}$ A grain was defined by the presence of continuous boundary-boundary exceeding $15^{\circ}$ misorientation.
} 
of GAM, towards higher GAM values, at the intermediate deformation temperature(s).

To outline the trends in GAM shifts, Fig. 2 and Table 2 summarizes the observations on average GAM at all deformation conditions. Such observations, for both $\gamma$-fiber and $\alpha$-fiber grains/orientations, bring out remarkably different trends:

- $40 \%$ deformation: no clear trend with deformation temperature.

- $60 \%$ deformation: GAM dropped with increase in working temperature.

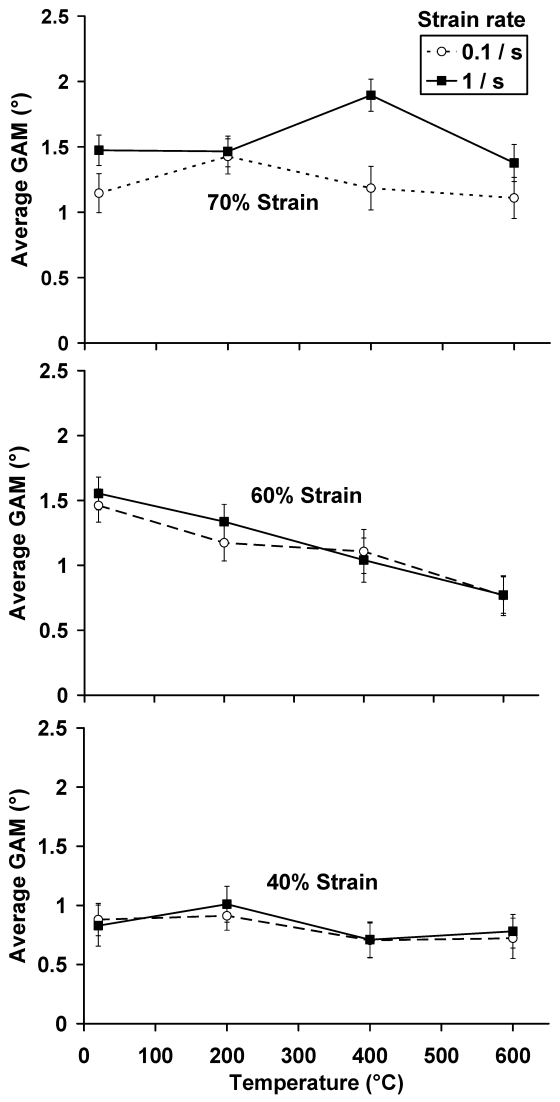

(a)
- 70\% deformation: an increase in GAM at intermediate $\left(200-400^{\circ} \mathrm{C}\right)$ deformation temperatures.

To comprehend the rationale behind such trends, visualization of the actual EBSD images are necessary. Figure 3 shows the images of two EBSD scans from the extreme deformation conditions - $40 \%$ strain at $600^{\circ} \mathrm{C}$ (Fig. 3(a)) and $70 \%$ strain at $20^{\circ} \mathrm{C}$ (Fig. 3(b)), both deformed at a strain rate of $0.1 \mathrm{~s}^{-1}$. The interesting feature in any such EBSD scans, also described ${ }^{10,17-23)}$ repeatedly in the domain of published literature, is the relative presence of grain interior strain localizations.

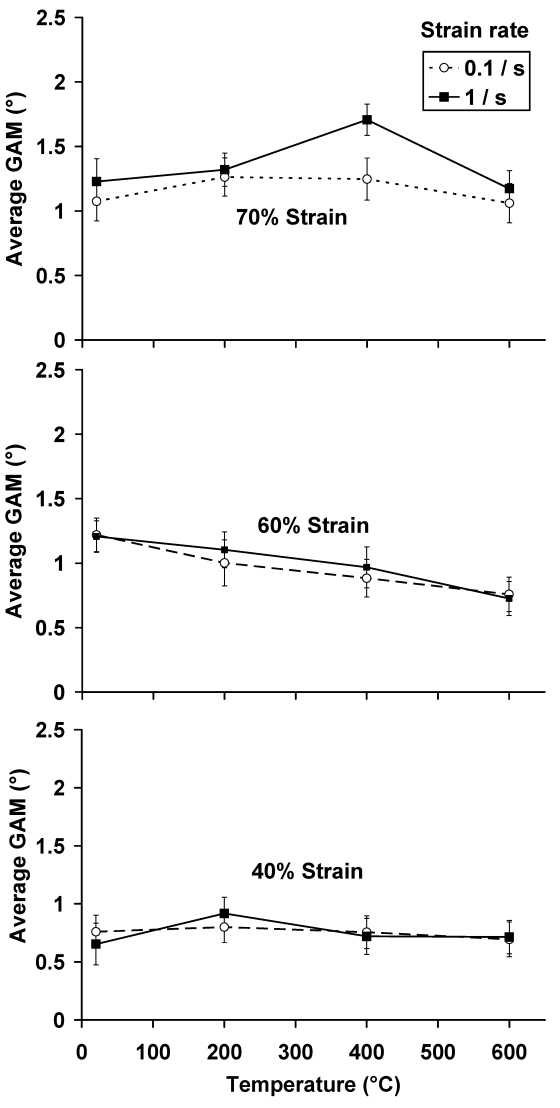

(b)

Fig. 2. Average GAM values at different deformation conditions for (a) $\gamma$-fiber and (b) $\alpha$-fiber grains/orientations.

Table 2. Average GAM values for $\gamma$-fiber and $\alpha$-fiber at different deformation conditions. The standard deviations are given in '( )'. The initial (fully recrystallized) structure had a $0.27^{\circ} \mathrm{GAM}$ and the same can be taken as measurement uncertainty.

\begin{tabular}{|c|c|c|c|c|c|}
\hline \multicolumn{3}{|c|}{$\gamma$-fiber (ND $/ /<111>$ ) } & \multicolumn{3}{|c|}{$\alpha$-fiber (RD // <110>) } \\
\hline${ }^{\circ} \mathrm{C}$ & $70 \%-0.1$ & $70 \%-1$ & ${ }^{\circ} \mathbf{C}$ & $70 \%-0.1$ & $70 \%-1$ \\
\hline 20 & $1.146(0.15)$ & $1.474(0.11)$ & 20 & $1.075(0.15)$ & $1.228(0.18$ \\
\hline 200 & $1.427(0.13)$ & $1.465(0.12)$ & 200 & $1.263(0.15)$ & $1.320(0.13$ \\
\hline 400 & $1.184(0.17)$ & $1.895(0.12)$ & 400 & $1.247(0.16)$ & $1.707(0.12$ \\
\hline 600 & $1.109(0.16)$ & $1.377(0.14)$ & 600 & $1.061(0.15)$ & $1.173(0.14$ \\
\hline
\end{tabular}

\begin{tabular}{|c|c|c|c|c|c|}
\hline \multicolumn{3}{|c|}{$\gamma$-fiber (ND //<111>) } & \multicolumn{3}{c|}{$\alpha$-fiber (RD //<110>) } \\
\hline${ }^{\circ} \mathbf{C}$ & $\mathbf{6 0 \% - 0 . 1}$ & $\mathbf{6 0 \% - 1}$ & ${ }^{\circ} \mathbf{C}$ & $\mathbf{6 0 \% - 0 . 1}$ & $\mathbf{6 0 \% - \mathbf { 1 }}$ \\
\hline $\mathbf{2 0}$ & $1.461(0.13)$ & $1.554(0.13)$ & $\mathbf{2 0}$ & $1.219(0.13)$ & $1.207(0.12)$ \\
\hline $\mathbf{2 0 0}$ & $1.174(0.14)$ & $1.336(0.14)$ & $\mathbf{2 0 0}$ & $1.002(0.18)$ & $1.104(0.14)$ \\
\hline $\mathbf{4 0 0}$ & $1.107(0.17)$ & $0.771(0.17)$ & $\mathbf{4 0 0}$ & $0.883(0.14)$ & $0.726(0.16)$ \\
\hline $\mathbf{6 0 0}$ & $0.767(0.15)$ & $1.040(0.14)$ & $\mathbf{6 0 0}$ & $0.758(0.13)$ & $0.968(0.13)$ \\
\hline
\end{tabular}

\begin{tabular}{|c|c|c|c|c|c|}
\hline \multicolumn{3}{|c|}{$\gamma$-fiber (ND //<111>) } & \multicolumn{3}{c|}{$\alpha$-fiber (RD //<110>) } \\
\hline${ }^{\circ} \mathbf{C}$ & $\mathbf{4 0 \% - \mathbf { 0 . 1 }}$ & $\mathbf{4 0 \% - 1}$ & ${ }^{\circ} \mathbf{C}$ & $\mathbf{4 0 \% - \mathbf { 0 . 1 }}$ & $\mathbf{4 0 \% - 1}$ \\
\hline $\mathbf{2 0}$ & $0.88(0.14)$ & $0.828(0.17)$ & $\mathbf{2 0}$ & $0.759(0.14)$ & $0.654(0.18)$ \\
\hline $\mathbf{2 0 0}$ & $0.912(0.12)$ & $1.010(0.15)$ & $\mathbf{2 0 0}$ & $0.8(0.13)$ & $0.917(0.14)$ \\
\hline $\mathbf{4 0 0}$ & $0.704(0.15)$ & $0.710(0.15)$ & $\mathbf{4 0 0}$ & $0.756(0.14)$ & $0.72(0.15)$ \\
\hline $\mathbf{6 0 0}$ & $0.722(0.17)$ & $0.781(0.14)$ & $\mathbf{6 0 0}$ & $0.694(0.15)$ & $0.714(0.15)$ \\
\hline
\end{tabular}




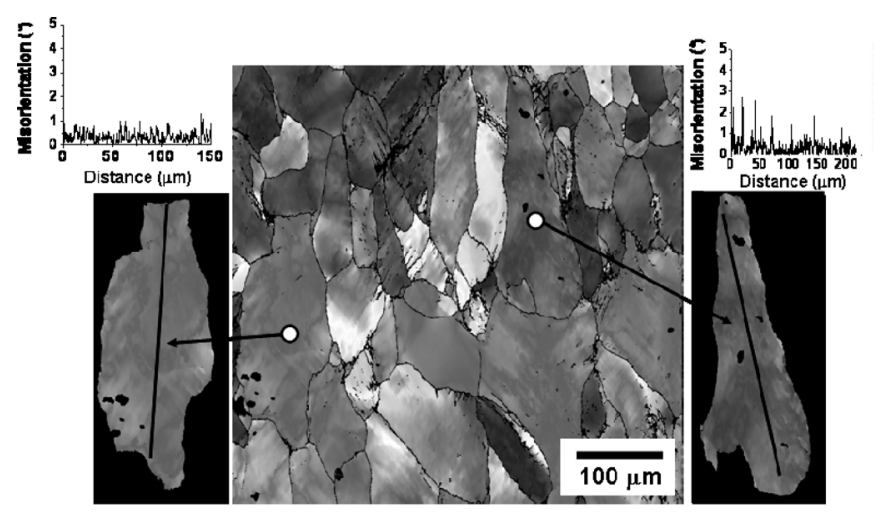

(a)
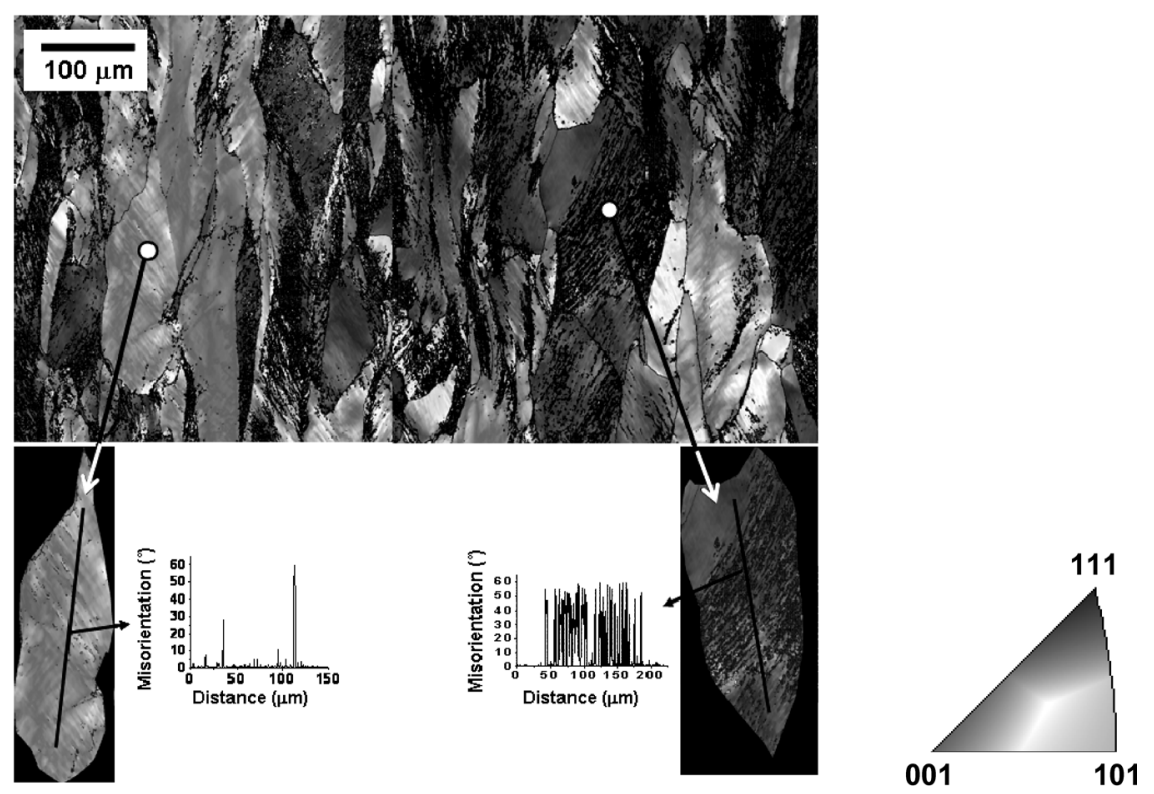

(b)

Fig. 3. Cropped $\gamma$-fiber and $\alpha$-fiber grains from larger EBSD maps for sample deformed at (a) $600^{\circ} \mathrm{C}-40 \%$ strain (b) $20^{\circ} \mathrm{C}-70 \%$ strain - both at strain rate of $0.1 \mathrm{~s}^{-1}$. Above $1^{\circ}$ grain boundaries are marked as black lines. In-grain misorientation developments in the cropped grains are also indicated.

The strain localizations in the individual pancaked grains can be 'viewed', qualitatively, ${ }^{* 3}$ by drawing misorientation profiles_-see Fig. 3. It was, however, considered necessary to quantify the relative 'presence' of grain interior strain localizations with respect to deformation conditions. The common problem in such quantification is to apply an appropriate logic for grain partitioning. In an automated EBSD analysis, grains are typically determined from the presence of pre-defined continuous grain boundaries-i.e. boundaries above certain misorientation. In heavily deformed grains, as in Fig. 3(b), such boundaries often do not match with visible appearance of a typical pancaked grain. The solution exists only in manual cropping of individual grains from the entire EBSD scan using appropriate user judgement. As shown in Fig. 3, from the EBSD scans visible $\gamma$-fiber and $\alpha$-fiber grains were cropped manually. This, on the other hand, allowed effective post-processing of
3800 grains, both $\gamma$-fiber and $\alpha$-fiber, for 24 deformation conditions.

On each cropped grain, 4 misorientation profiles (on average) were taken - at random and along the rolling direction. From such misorientation profiles (approximately 15200 profiles), data were extracted for number of high angle (i.e. above $15^{\circ}$ misorientation) boundaries and for average misorientation. For the later, misorientation in excess of $1^{\circ}$ (i.e. beyond measurement uncertainty ${ }^{41)}$ ) was considered. ${ }^{*}$ Estimated average misorientation involving cropped grains has similarities with automated GAM estimates (Figs. 1, 2 and Table 2), though the former does not suffer from difficulties in grain definition. Figure 4 provides the trends in the number of $>15^{\circ}$ boundaries (per $100 \mu \mathrm{m}$ length of misorientation profile); while Fig. 5 outlines the patterns on average misorientation. For readers convenience/reference Table 3 collates the data on bound-

\footnotetext{
${ }^{*} \gamma$-fiber grains had more strain localizations. However, exact differences, especially differences as a function of deformation conditions, need further (and more detailed) quantification.

${ }^{* 4}$ It needs to be noted that almost always such misorientations are associated with visible strain localizations. This point/observation has also been reported in past $^{18-20)}$ publications.
} 

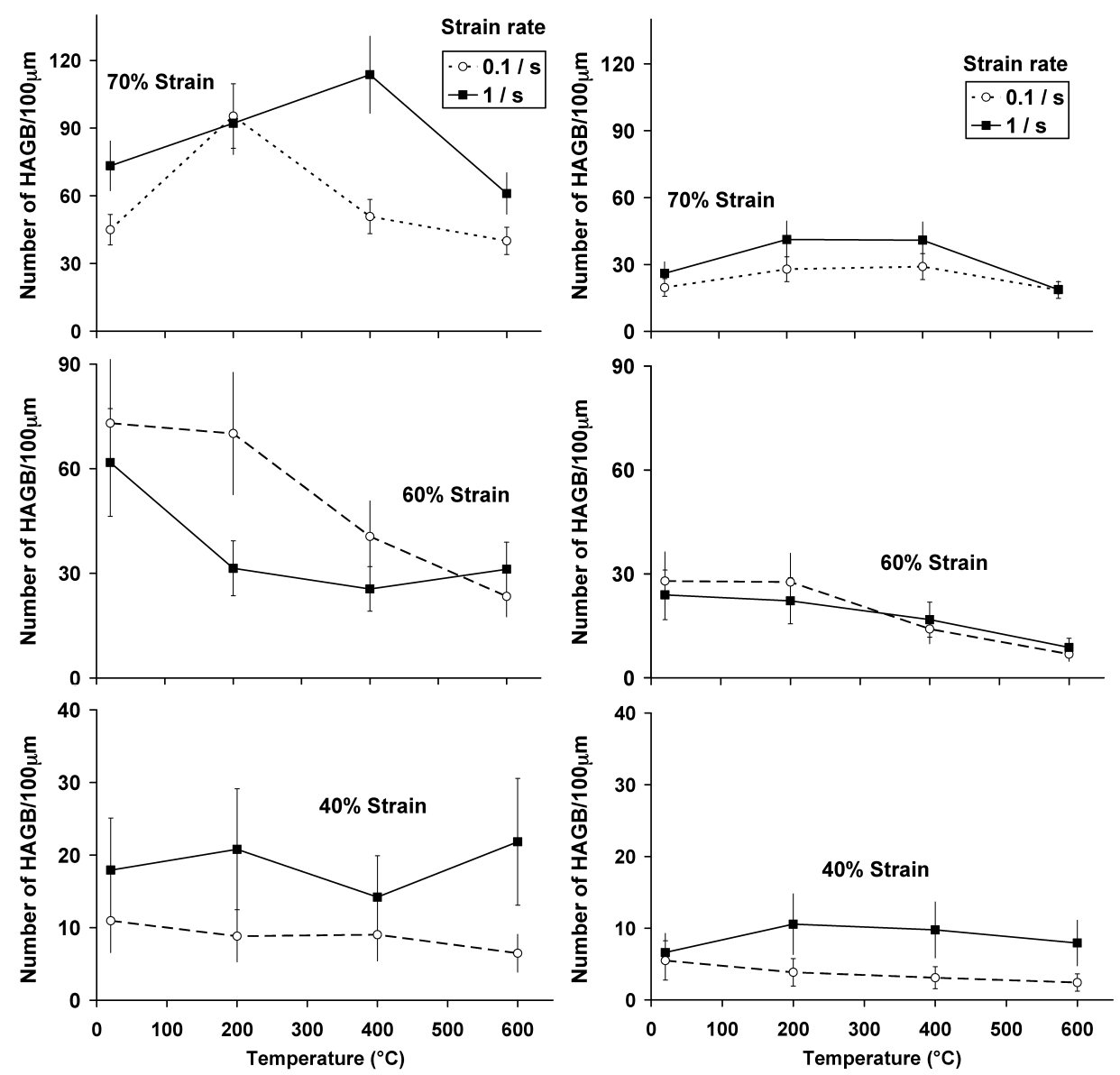

(a)

(b)

Fig. 4. Number of high angle boundaries (HAGB) per $100 \mu \mathrm{m}$ length of the individual/cropped grains. Data are represented for different deformation conditions and for (a) $\gamma$-fiber and (b) $\alpha$-fiber grains/orientations.

ary fractions (of specified misorientation) at different deformation conditions.

It is interesting to note that the tedious data extraction/ analysis, Figs. 4 and 5 and Table 3, through manual cropping, yielded a trend nearly identical to the trends in average GAM (Figs. 1, 2 and Table 2). In other words, increased GAM at the intermediate temperatures (200$400^{\circ} \mathrm{C}$ ) is clearly due to increased (both in number and also in misorientation) presence of grain-interior strain localizations.

\section{Discussion}

The experimental observations, as discussed in the earlier section, have two main points:

- An apparent high in in-grain misorientation (GAM) after $70 \%$ deformation in the intermediate $\left(200-400^{\circ} \mathrm{C}\right)$ temperature range.

- This was caused by an increased presence, especially in $\gamma$-fiber grains, of grain-interior strain localizations.

The present section aims at rationalizing these observations in terms of dynamic strain aging, ${ }^{42-53)}$ recovery ${ }^{40)}$ and instability criteria. $^{24-27)}$

In low carbon steel, there are two usual approaches explaining temperature/orientation dependence of strain localizations - explanations through dynamic strain aging $(\mathrm{DSA})^{50-53)}$ and/or from instability criteria. ${ }^{24-27)}$ DSA represents dynamic interactions between mobile dislocations and solute (e.g. carbon and nitrogen in ULC steel) atoms. This may give rise to abnormally low or even negative ${ }^{50-53)}$ strain rate sensitivities resulting in flow localization. The peak of this effect, under the strain rates used in the present study, is expected ${ }^{54,55)}$ to fall in the range from 150 to $400^{\circ} \mathrm{C}$ - and in turn may explain, albeit qualitatively, the observed temperature dependence. There are, however, specific problems with a DSA explanation:

- Though temperature dependence of strain rate sensitivity has often been reported, ${ }^{50-53)}$ the present study does not reveal clinching experimental evidence for DSA in the present ULC steel.

- It is not too apparent why effects of DSA on flow localization were felt only at $70 \%$ reduction and not at the lower reductions.

- Orientation dependence of DSA is also difficult to comprehend - other than a loose argument on orientation dependence of solute-dislocation interactions.

An explanation through instability criteria, on the other hand, can explain the orientation as well as temperature dependence of grain interior strain localizations. A classical, and much talked about, instability criteria is the Dillamore's criterion $^{24)}$ or Eq. (1). Dillamore himself had used this criterion to link crystallographic and morphological orientations of fcc shear bands. ${ }^{24}$ It has also been used by subsequent researchers to explain formation of strain localizations in a variety of materials, ${ }^{19-22,25-27,56,57)}$ including low carbon steel. Dillamore's instability criterion, Eq. (1), calls for 

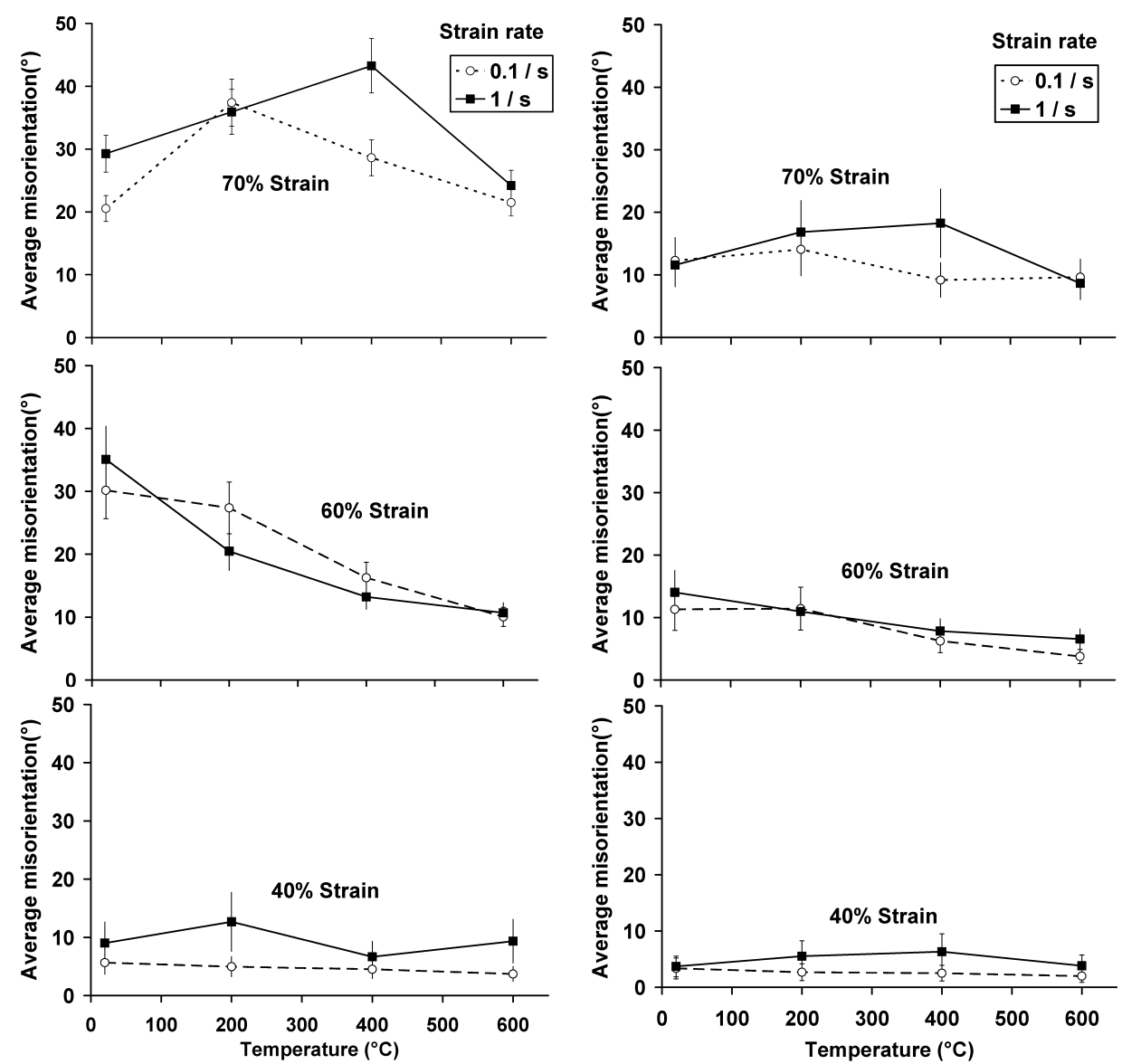

(a)

(b)

Fig. 5. Average misorientation arising from grain-interior strain localizations — visible (as in Fig. 4) bands of above $1^{\circ}$ misorientation. Data were obtained from individual/cropped grains and are represented for different deformation conditions and for (a) $\gamma$-fiber and (b) $\alpha$-fiber grains/orientations.

Table 3. Number fraction of boundaries (of specified misorientation range) inside $\gamma$-fiber and $\alpha$-fiber grains. The data were obtained from cropped grains of the respective fibers.

\begin{tabular}{|c|c|c|c|c|c|c|c|c|c|c|c|c|c|}
\hline \multirow[b]{3}{*}{${ }^{\circ} \mathrm{C}$} & \multicolumn{6}{|c|}{$\gamma$-fiber (ND//<111>) } & & \multicolumn{6}{|c|}{$\alpha$-fiber (RD//<110>) } \\
\hline & \multicolumn{3}{|c|}{$70 \%-0.1$} & \multicolumn{3}{|c|}{$70 \%-1$} & & \multicolumn{3}{|c|}{$70 \%-0.1$} & \multicolumn{3}{|c|}{$70 \%-1$} \\
\hline & 1 to $5^{\circ}$ & 5 to $15^{\circ}$ & $>15^{\circ}$ & 1 to $5^{\circ}$ & 5 to $15^{\circ}$ & $>15^{\circ}$ & ${ }^{\circ} \mathrm{C}$ & 1 to $5^{\circ}$ & 5 to $15^{\circ}$ & $>15^{\circ}$ & 1 to $5^{\circ}$ & 5 to $15^{\circ}$ & $>15^{\circ}$ \\
\hline 20 & 0.540 & 0.065 & 0.394 & 0.396 & 0.092 & 0.510 & 20 & 0.713 & 0.069 & 0.217 & 0.613 & 0.143 & 0.243 \\
\hline 200 & 0.290 & 0.030 & 0.680 & 0.342 & 0.093 & 0.564 & 200 & 0.586 & 0.031 & 0.382 & 0.489 & 0.213 & 0.297 \\
\hline 400 & 0.437 & 0.043 & 0.518 & 0.240 & 0.080 & 0.678 & 400 & 0.662 & 0.063 & 0.274 & 0.559 & 0.057 & 0.382 \\
\hline 600 & 0.501 & 0.057 & 0.4411 & 0.393 & 0.138 & 0.467 & 600 & 0.696 & 0.055 & 0.247 & 0.688 & 0.085 & 0.226 \\
\hline
\end{tabular}

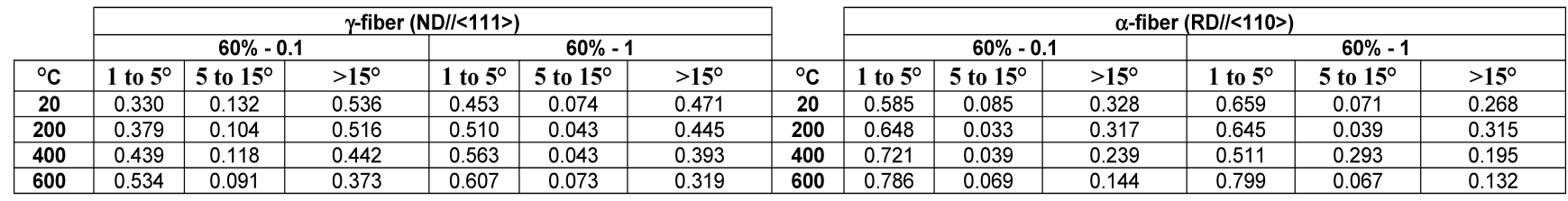

\begin{tabular}{|c|c|c|c|c|c|c|c|c|c|c|c|c|c|}
\hline \multirow[b]{3}{*}{${ }^{\circ} \mathrm{C}$} & \multicolumn{6}{|c|}{$\gamma$-fiber (ND//<111>) } & & \multicolumn{6}{|c|}{$\alpha$-fiber $(\mathrm{RD} / /<110>$ ) } \\
\hline & \multicolumn{3}{|c|}{$40 \%-0.1$} & \multicolumn{3}{|c|}{$40 \%-1$} & & \multicolumn{3}{|c|}{$40 \%-0.1$} & \multicolumn{3}{|c|}{$40 \%-1$} \\
\hline & 1 to $5^{\circ}$ & 5 to $15^{\circ}$ & $>15^{\circ}$ & 1 to $5^{\circ}$ & 5 to $15^{\circ}$ & $>15^{\circ}$ & ${ }^{\circ} \mathrm{C}$ & 1 to $5^{\circ}$ & 5 to $15^{\circ}$ & $>15^{\circ}$ & 1 to $5^{\circ}$ & 5 to $15^{\circ}$ & $>15^{\circ}$ \\
\hline 20 & 0.728 & 0.051 & 0.220 & 0.628 & 0.034 & 0.336 & 20 & 0.819 & 0.038 & 0.142 & 0.622 & 0.199 & 0.178 \\
\hline 200 & 0.779 & 0.052 & 0.168 & 0.641 & 0.031 & 0.326 & 200 & 0.841 & 0.045 & 0.113 & 0.626 & 0.205 & 0.168 \\
\hline 400 & 0.717 & 0.032 & 0.249 & 0.649 & 0.036 & 0.313 & 400 & 0.886 & 0.031 & 0.081 & 0.507 & 0.257 & 0.234 \\
\hline 600 & 0.768 & 0.036 & 0.194 & 0.560 & 0.022 & 0.417 & 600 & 0.865 & 0.041 & 0.093 & 0.574 & 0.223 & 0.202 \\
\hline
\end{tabular}

plastic instabilities in cases of negative values of texture softening $(d M / d \varepsilon)$ and/or positive values of dislocation softening $(d \rho / d \varepsilon)$. Past research ${ }^{27)}$ had shown clear link between presence of strain localizations and estimated $d M / d \varepsilon$. It was also shown, ${ }^{27)}$ albeit theoretically, that the crystallographic orientations with high Taylor factor $(M)$ are often the orientations with a greater tendency to rotate to softer orientations and hence they represent negative textural softening. It may be noted that, in general, $\gamma$-fiber and $\alpha$-fiber grains represent high and low Taylor factors ${ }^{2,10,13,58,59)}$ and are correspondingly orientations with negative and positive textural softening. This, on other hand, explains the observed orientation dependence of grain interior strain localizations. 
Dillamore's criterion also depends on dislocation softening or $d \rho / d \varepsilon$. Arguably, part of this can also be orientation sensitive. This needs to be viewed in terms of both deformation and recovery-demarcating them during warm working being a nearly impossible task. In experimental observations of static recovery a drop in misorientation is usually expected. ${ }^{40)}$ The only exception, attributed to the effects of texturing, is past observation from the Trondheim group, ${ }^{60)}$ where a clear increase in misorientation with static recovery has been reported. Strong recovery with deformation can, however, 'enhance' the presence of plastic instabilities $^{59,61)}$ and a corresponding increase ${ }^{60)}$ in misorientation developments. This would also depend on the values of strain and of strain hardening exponent $n$. For example, higher strain is typically expected ${ }^{40)}$ to bring stronger recovery kinetics; while an expected drop in $n$ during high temperature deformation ${ }^{10)}$ may cancel out beneficial effects of recovery on the formation of strain localizations.

If one considers a 'balance' between textural and dislocation softening (e.g. Dillamore's inequality-Eq. (1)), it is apparent that if the later is negligible then the orientations with negative textural softening should have stronger preference for strain localizations. $d \rho / d \varepsilon$ can be reduced, with corresponding enhancement of role/effects of $d M / d \varepsilon$, by (i) lowering solute atoms and/or (ii) higher deformation temperature. The first forms the basis of low carbon (or low interstitial) steels as the dominant class of today's forming grade material ${ }^{2,8-10,62-64)}$; while the present study provides clear indications about the success of warm rolling as an effective means for enhancing the effects of $d M / d \varepsilon$.

More specifically, and with emphasis on the present set of results, it appears that Dillamore's criterion offers an overall, albeit qualitative, explanation. Quantities like $n$ and $m$ are expected to be affected by working temperature - an effect linked to the misorientation developments for $n$ and solute-dislocation interactions for $m$. Both are expected to drop with increase in deformation temperature. This is a general 'expectation'; though an elevated temperature DSA and/or misorientation developments through creation of strain localizations can alter such trends. The two most important entities of Dillamore's inequality are, however, textural $(d M / d \varepsilon)$ and dislocation $(d \rho / d \varepsilon)$ softening. $d M / d \varepsilon$ can be hypothesized to be sensitive only to crystallographic orientation. It needs to be noted that the crystallographic texture was not affected, significantly, by the range of deformation temperature and strain rate, used in the present study, and correspondingly a generalization on the insensitivity of $d M / d \varepsilon$ on temperature and strain rate is hypothesized/proposed. ${ }^{5}$ In other words, if $d M / d \varepsilon$ did not get affected, at least significantly, by the deformation conditions; then the observed patterns of GAM developments (Fig. 2) and corresponding differences in relative presence of strain localizations (Figs. 4, 5) were caused primarily by $d \rho / d \varepsilon$. A simplistic view will be reduced $d \rho / d \varepsilon$, through recovery, and corresponding enhancement of the role of $d M / d \varepsilon$. This can, in theory, enhance preferred or orientation sensitive formation of strain localizations, provided a very strong recovery does not lower $n$ dramatically. But a monotonic drop in $d \rho / d \varepsilon$, insensitive of strain and crystallo- graphic orientation, cannot explain: (i) an apparent 'high' in GAM after $70 \%$ (and not $60 \%$ ) deformation and (ii) the effects being significantly more in the deformed $\gamma$-fiber grains. Role of strain on recovery and of possible orientation sensitivity of recovery can explain such results, but they remain 'undefined' at this stage and should formulate directions for future research. It also remains to be charted how far the present observations can be translated into sustainable engineering advantage, but the present study clearly brings out the possibility of - optimized stored energy advantage for deformed $\gamma$-fiber through controlled warm working.

\section{Conclusions}

(1) At the highest strain, 70\% reduction in thickness, in-grain misorientation development had shown a clear 'high' for the intermediate $\left(200-400^{\circ} \mathrm{C}\right)$ deformation temperature. This effect, more apparent for deformed $\gamma$-fiber, was caused by an increased presence of grain interior strain localizations.

(2) The effect can be explained from possibly dynamic strain aging or a balance between textural $(d M / d \varepsilon)$ and dislocation $(d \rho / d \varepsilon)$ softening - a balance ultimately determining a micro-scale instability criterion. Whatever is the possible explanation and technological implications, the present study clearly brings out-optimized stored energy advantage for deformed $\gamma$-fiber through controlled warm working.

\section{Acknowledgement}

The authors would like to acknowledge the use of the National Facility of Texture and OIM (a DST-IRPHA facility) for this study. Financial support from IFCPAR/CEFIPRA (Indo-French Centre for the promotion of advance research) and DST (Department of Science and Technology) are also acknowledged.

\section{REFERENCES}

1) S. Mishra and C. Darmann: Int. Met. Rev., 27 (1982), No. 6, 307.

2) W. B. Hutchinson: Int. Met. Rev., 29 (1984), No. 1, 25.

3) J. S. Kallend and C. S. Da Costa Viana: Proc. on Formability and Metallurgical Structure, ed. by A. K. Sachdev and J. D. Embury, The Metallurgical Society, Warrendale, PA, (1986), 137.

4) W. B. Hutchinson and L. Ryde: 16th Risø Symp. on Mater. Sci., ed. by Hansen, D. Juul Jensen, Y. L. Liu and B. Ralph, Risø National Lab, Roskilde, Denmark, (1995), 105.

5) W. B. Hutchinson and E. Lindh: Int. Forum on Phys. Met. in IF Steel, ISIJ, Tokyo, (1994), 127.

6) Y. Hayakawa and J. A. Szpunar: Acta Mater, 45 (1997), No. 6, 2425.

7) J. J. Jonas and T. Urabe: Int. Forum on Phys. Met. in IF Steel, ISIJ, Tokyo, (1994), 143.

8) K. Ito: Forum on Phys. Met. in IF Steel, ISIJ, Tokyo, (1994), 99.

9) R. K. Ray, J. J. Jonas and R. E. Hook: Int. Mater. Rev., 39 (1994), No. 4,129

10) B. Verlinden, J. Driver, I. Samajdar and R. D. Doherty: Thermo-Mechanical Processing of Metallic Materials, ISBN-978-0-08-0444970, Pergamon Materials Series_-series ed. by R. W. Cahn, Elsevier, Amsterdam, (2007).

11) L. Kestens and J. J. Jonas: ISIJ Int., 37 (1997), No. 8, 807.

12) S. H. Hong and D. N. Lee: ISIJ Int., 42 (2002), No. 11, 1278.

13) L. Kestens and J. J. Jonas: Metall. Mater. Trans. A, 27A (1996), 155.

14) L. Kestens and J. J. Jonas: Metall. Mater. Trans. A, 27A (1996),

\footnotetext{
${ }^{* 5}$ It also needs to be noted that the crystallographic texture was largely stable beyond $60 \%$ deformation.
} 
2347.

15) F. Emren, U. von Schlippenbach and K. Lücke: Acta Metall., 34 (1986), 2105.

16) Y. B. Park, D. N. Lee and G. Gottstein: 16th Riso Symp. on Mater. Sci., ed. by N. Hansen, D. Juul Jensen, Y. L. Liu and B. Ralph, Riso National Lab, Roskilde, Denmark, (1995), 479.

17) K. Ushioda and W. B. Hutchinson: ISIJ. Int., 29 (1989), No. 10, 862.

18) G. H. Akbari, C. M. Sellars and J. A. Whiteman: Acta Mater, 45 (1997), No. 12, 5047.

19) V. M. Nandedkar, I. Samajdar and K. Narashiman: ISIJ Int., 41 (2001), No. 12, 1517

20) I. Samajdar, B. Verlinden, L. Kestens and P. Van Houtte: Acta Mater., 47 (1999), No. 1, 55.

21) T. Haratani, W. B. Hutchinson, I. L. Dillamore and P. Bate: Met. Sci., 18 (1984), 57.

22) I. Samajdar, B. Verlinden and P. Van Houtte: Acta Mater, 46 (1998), No. 8, 2751.

23) B. L. Li, A. Godfrey, Q. C. Meng, Q. Liu and N. Hansen: Acta Mater, 52 (2004), 1069.

24) I. L. Dillamore, J. G. Roberts and A. C. Bush: Met. Sci., 13 (1979), 73

25) J. Gil Sevillano, P. Van Houtte and E. Aernoudt: Prog. Mater. Sci., 25 (1980), 69.

26) P. Wagner, O. Engler and K. Lücke: Acta Metall. Mater., 43 (1995), No. 10,3799

27) S. Cicalè, I. Samajdar, B. Verlinden, G. Abbruzzese and P. Van Houtte: ISIJ Int., 42 (2002), 770.

28) OIM Analysis Version 4.5, User Manual: Tex Sem laboratories Inc., Draper, UT, USA, (2004).

29) B. Bay, N. Hansen, D. A. Hughes and D. Kuhlmann-Wilsdorf: Acta Metall., 40 (1992), 205.

30) H. Weiland: Acta Mater, 40 (1992), 1083

31) J. A. Wert, Q. Liu and N. Hansen: Acta Metall., 43 (1995), 4153.

32) D. A. Hughes, Q. Liu, D. C. Chrzan and N. Hansen: Acta Mater, 45 (1997), 105

33) M. D. Nave and M. R. Barnett: ISIJ Int., 44 (2004), 187.

34) B. Hutchinson, N. Hansen, P. Van Houtte and D. Juul Jensen: Philos. Trans. R. Soc. (London), A357 (1999), 1471.

35) D. Vanderschueren, N. Yoshinaga and K. Koyama: ISIJ Int., 36 (1996), 1046.

36) B. J. Duggan, M. Hatherly, W. B. Hutchinson and P. T. Wakefield: Met. Sci., 12 (1978), 343.

37) J. Jensen: Mater. Sci. Eng. A, A234-236 (1997), 1170.

38) G. Winther and X. Huang: Adv. Eng. Mater, 5 (2003), 317.

39) J. Ch. Glez and J. H. Driver: Acta Mater., 51 (2003), 2989.

40) F. J. Humphreys and M. Hatherly: Recrystallization and Related An- nealing Phenomena, Elsevier Science Limited, UK, (1995), 11.

41) S. K. Sahoo, V. D. Hiwarkar, I. Samajdar, G. K. Dey, D. Srivastav, R. Tiwari and S. Banerjee: Scr. Mater., 56 (2007), 963.

42) P. Hahner: Mater. Sci. Eng. A, A164 (1993), 23.

43) P. Hahner and R. Egidio: Acta Mater, 51 (2003), 3385.

44) K. Chihab, Y. Estrin, L. P. Kubin and J. Vergnol: Scr. Metall., 21 (1987), 203

45) P. G. McCormick: Acta Metall., 36 (1988), 3061.

46) Y. Estrin and L. P. Kubin: Continuum Models for Materials with Microstructures, ed. by, H. B. Muhlhaus, Wiley, New York, (1995), 395.

47) D. J. Lloyd, S. A. Court and K. M. Gatenby: Mater. Sci. Technol., 13 (1997), 660

48) Z. Kovacs, J. Lendvai and Voros: Mater. Sci. Eng. A, A279 (2000), 179.

49) Z. A. Chmelik, Neuhauser and Luckac: Mater. Sci. Eng. A, A324 (2002), 200

50) M. R. Barnett: ISIJ Int., 38 (1998), No. 1, 78.

51) M. R. Barnett and J. J. Jonas: ISIJ Int., 37 (1997), 697.

$52)$ D. Liu, A. O. Humphreys, M. R. Toroghinejad and J. J. Jonas: ISIJ Int., 42 (2007), No. 7, 751.

53) A. O. Humphreys, D. Liu, M. R. Toroghinejad, E. Essadiqi and J. J. Jonas: Met. Sci. Techro., 19 (2003), 709.

54) J. D. Baird and A. Jamieson: J. Iron Steel Inst., (1966), 793.

55) S. L. Semiatin and J. J. Jonas: Formability and Workability of Metals-Plastic Instability and Flow Localization, ASM, Ohio, (1984), 93.

56) I. Samajdar, P. Ratchev, B. Verlinden, P. Van Houtte and P. De Smet: Mater. Sci. Eng. A, A247 (1998), 58.

57) M. Kiran Kumar, C. Vanitha, I. Samajdar, G. K. Dey, R. Tewari, D. Srivastava and S. Banerjee: Mater. Sci. Technol., 22 (2006), 331.

58) N. Rajmohan, Y. Hayakawa, J. A. Szpunar and J. H. Root: Acta Mater, 45 (1997), 2485.

59) I. Samajdar, B. Verlinden, P. Van Houtte and D. Vanderschueren: Mater. Sci. Eng. A, A238 (1997), 343.

60) G. J. Baxter, T. Furu, Q. Zhu, J. A. Whiteman and C. M. Sellars: Acta Mater., 47 (1999), 2367.

61) A. Korbel, J. D. Embury, M. Hatherly, P. L. Martin and H. W. Erbsloh: Acta Metall., 10 (1986), 1999.

62) J. J. Jonas: Modern LC and ULC Sheet Steels for Cold Forming: Processing and Properties, ed. by W. Bleck, Verlag Mainz, Aachen, (1998), 73.

63) J. S. Kallend and C. S. Da Costa Viana: Proc. on Formability and Metallurgical Structure, ed. by A. K. Sachdev and J. D. Embury, The Metallurgical Society, Warrendale, PA, (1986), 137.

64) M. R. Barnett and J. J. Jonas: ISIJ Int., 39 (1999), 856. 